\title{
Entwicklungszusammenarbeit in Zentralafrika
}

Bemerkungen und Überlegungen nach einem dreijährigen Feldeinsatz als Koordinator der Projekte des Dienstes für Entwicklungszusammenarbeit und humanitäre Hilfe (DEH) in Kamerun und Tschad.

\section{Ausgangslage}

Unsere Entwicklungszusammenarbeit ist noch sehr jung. Erst zwanzigjährig! Glücklicherweise - so ist festzustellen - hat sie noch kein einheitliches Modell entwickelt, keine unabänderlichen Vorgehen definiert.

Ein Bundesgesetz regelt Zweck und Zielsetzungen 1); innerhalb dieser Vorgabe jedoch gilt es, die Entwicklungsprogramme und -projekte mit großer Flexibilität an die international und intranational ganz verschiedenen Verhältnisse in der 3. Welt anzupassen. Die Realität zeigt, daß dies - auch durch eine Bundesverwaltung - durchaus möglich ist.

Die Anfragen zur Unterstützung einer Entwicklungsaktion gehen von ganz verschiedenen Organisationen und Sachbereichen aus, wobei die politische Situation, die vorhandenen Ressourcen, materiellen Mittel, personellen Möglichkeiten usw. sehr unterschiedlich sind. Meist gilt es deshalb, für jedes Projekt einen eigenen Weg zu suchen; Fehler, Überprüfungen und Neuausrichtungen sind häufig, Transpositionen von Erfahrungen üblicherweise nur in Teilbereichen möglich. Die Ausnahme bildet die Regel! Projektthematik, Aktivitätssektoren und Vorgehensmethodik sind infolgedessen ebenfalls sehr differenziert. Eine Koordinationstätigkeit bleibt deshalb notwendigerweise in Generalistenaufgaben stecken und ermangelt oft der Tiefe; die Fülle von Aufgaben fasziniert jedoch durch ihre Vielfältigkeit.

Dieser verwirrende Eindruck von wenig kohärenten Einzelaspekten, die noch nicht zu klaren Gesamtkonturen gefunden haben, entstand auch bei mir zu Beginn meiner Koordinationstätigkeit in Kamerun und Tschad, zwei Schwerpunktländern der schweizerischen Entwicklungszusammenarbeit, wo die DEH in den letzten Jahren ca. 2 Millionen Franken pro Jahr und Land sowie insgesamt an die 30 Experten einsetzte.

1) Vgl. Bericht H. Escher: Zur Tätigkeit bei der DEH.
Das Aktionsprogramm in diesen beiden Ländern ist sehr pragmatisch gewachsen: Missionen, die zum Teil bereits seit Jahrzehnten in Zentralafrika wirkten, baten die Eidgenossenschaft um Unterstützung für ihre Entwicklungstätigkeit: Grundschulung, medizinische Versorgung und etwas praktische landwirtschaftliche und technische Ausbildung standen dabei im Vordergrund. Häufig wurden junge Freiwillige eingesetzt, die mit sehr viel gutem Willen und altruistischer Einstellung mitarbeiteten. Es war die «Pfadfinderzeit» der Entwicklungshilfe, Idealismus und Irrealismus oft vereint. Verschiedene solche Projekte laufen heute - mannigfach abgeändert weiter, ihre Einflußnahme auf das Milieu und die Bevölkerung kann, dank langfristiger Einwirkung, groß sein.

Das erste Entwicklungsjahrzehnt brachte dann den Glauben an den technischen Fortschritt und die Überzeugung einer möglichen Übertragung westlich-logischer Technik auf Entwicklungsländer. Infrastrukturprojekte, Vermittlung von Know-how, Kleinindustrien, Mechanisierung waren einige Stichworte, die zu Aktionen führten, die - wiederum angepaßt und abgeändert - teilweise auch heute noch weitergeführt werden.

Die letzten Jahre waren - mindestens was West- und Zentralafrika betrifft - von Seiten der Geberländer einerseits auf eine verstärkte Berücksichtigung sozio-kultureller Aspekte und damit einer Arbeit an der Basis in relativ bescheidener finanzieller Größenordnung ausgerichtet, anderseits auf die Realisierung von großen, komplexen Projekten im Bereich der Infrastrukturen sowie der Agroindustrie. Dieser letztere Aspekt wurde in voller Übereinstimmung mit den nationalen Entscheidungsstrukturen der Dritt-Welt-Länder bearbeitet, die meist zwischen 1965 und 1970 Entwicklungspläne einführten und damit staatliche Entwicklungsstrategien und Prioritäten festlegten.

Mit diesen Entwicklungsplänen stellte sich für die äußere Hilfe die Forderung nach einer Einpassung ihrer Tätigkeit in die staatlich fixierten Schwergewichte. Dies bedeutet, daß das Entwicklungsland

Dr. phil. nat. A. Bisaz, Morgenstraße 12, 3073 Gümligen. 
selbst bis zu einem gewissen Grad den Geberländern "vorschreibt», resp. suggeriert, welche Projekte sie doch realisieren möchten. Damit entstand wiederum eine andere Art des Vorgehens zur Auswahl von Projekten, die mit den bisherigen pragmatischen und praktischen Entscheidungen von Fall zu Fall nicht mehr in Einklang zu bringen war.

Das gegenwärtig laufende Entwicklungsprogramm der DEH in Kamerun oder Tschad spiegelt denn auch all diese Entwicklungs-Epochen wieder: Neben einfachen Basisprojekten mit einem wichtigen sozialen Aspekt stehen technisch anspruchsvolle Aktionen, neben finanziell sehr bescheidenen Unterstützungen lokaler Initiativen teure Infrastrukturaufgaben, neben praktischer Ausbildung theoretische Schulung, neben Förderung der Eigenversorgung im Nahrungsmittelbereich Unterstützungen für die Produktion von Cash-Crops usw.

Ein solches Multipack-Programm ist von seiner Entstehung her verständlich, es vermag aber angesichts der wachsenden qualitativen und quantitativen Ansprüche der Entwicklungsländer sowie der teilweisen Kontraproduktivität einzelner Projekte nicht mehr $\mathrm{zu}$ befriedigen. Auch stimmen gewisse Aktionen nicht oder nur ungenügend mit unserem Gesetz, den Entwicklungsausrichtungen der Staaten und unseren Absichten einer engen Zusammenarbeit mit der Bevölkerung überein.

In den letzten Jahren wurde deshalb in einigen unserer Schwerpunktländer der Versuch unternommen, unserer Entwicklungszusammenarbeit eine klarere Richtlinie zu geben, die den erwähnten Anforderungen besser entspricht. Allein die Definition eines gemeinsamen Nenners aller Anforderungen erwies sich dabei als sehr schwierig, ebenso die Diskussionen mit der Administration des Entwicklungslandes über schweizerische Entwicklungsvorstellungen und Vorschriften zur Kooperation. Auch gilt es zu erkennen, daß eine Anpassung an eine einmal erarbeitete Richtlinie sich über Jahre zu erstrecken hat, können doch laufende Projekte nicht ohne weiteres abgebrochen oder «übergeben» werden. Im Verlaufe der Jahre ändern aber wieder die Ansichten und Zielsetzungen, so daß auch eine Richtlinie evolutiv zu halten und kontinuierlich anzupassen ist.

Der Versuch der Ausarbeitung einiger Grundelemente für ein solches «Konzept» einer DEHUnterstützung der Entwicklung für Kamerun einerseits, für die Sahelregion (insbesondere den Tschad) anderseits, war eine der anspruchsvollsten Aufgaben meiner Tätigkeit in Zentralafrika, die daneben vor allem die Erbringung von Dienstleistungen sowie Überwachung und Betreuung für die laufenden Projekte, Verhandlungen mit den Behörden über die Realisierung der Aktionen, Studium und Ausarbeitung von Vorschlägen für neue Projekte sowie Unterstützung der Experten bei der Durchführung ihrer Aufgabe umfaßte.
Einige der Hauptelemente, die es neben den fixierten Punkten (Anforderungen des Bundesgesetzes, Entwicklungspläne des Landes usw.) für ein solches $\mathrm{Zu}$ sammenarbeitskonzept, resp. Landesprogramm der $\mathrm{DEH}$ für ein bestimmtes Land zu beachten gilt, seien nachfolgend kurz dargelegt.

\section{Definition der Hauptbedürfnisse}

Während für das «Wie» einer Entwicklungszusammenarbeit z. T. erprobte Vorgehensweisen bekannt sind, ist für das "Was», für die Bedürfnisfrage, oft eine eingehendere Abklärung notwendig.

Die vom Land in seinen Entwicklungsplänen definierten Bedürfnisse entsprechen meist den Ideen der Regierungsstellen (und ihrer europäischen Berater) und basieren auf einer europäisch-logischen Perzeption der Probleme; sie stimmen oft nicht oder höchstens teilweise mit den Aspirationen der Bevölkerung überein.

Als Beispiel sei die von der kamerunischen Regierung in den letzten Jahren stark geförderte Agroindustrie erwähnt. Sie mag dem Land zwar wohl Devisen, neue Arbeitsplätze, einen modernen Anstrich und eine Produktionsmaximierung bringen. Sie vergrößert aber gleichzeitig die Abhängigkeit Kameruns von den Multis sowie anderen Lieferanten von Agroinputs und Abnehmern der Produkte. Noch verstärkt wirkt sich diese Abhängigkeit auf den Landarbeiter aus, der ganz der halbstaatlichen Agrounternehmung «gehört», oder auf den Bauern, der meist nur der entsprechenden Unternehmung seine Produkte verkaufen kann. Ob dieses Vorgehen dem besten Entwicklungsweg entspricht, darf füglich bezweifelt werden.

Es gilt deshalb, auch von der Sicht der betroffenen Bevölkerung her die wichtigsten Bedürfnisse zu definieren. Diese spürt meist recht genau, wo ihre Probleme liegen; sie verfügt aber oft über zu wenig Kenntnisse, um ihre Wünsche "verwaltungsgemäß» zu definieren, über zu wenig Initiative, Mittel und Know-how, um eigene Schritte zur Lösung der Probleme einzuleiten und schließlich über wenig etablierte Strukturen, um ihre Anliegen bei den zuständigen Stellen mit dem nötigen Druck zu vertreten. Oft verhindern auch private Interessen etablierter Kreise jede Änderung der Verhältnisse.

Durch Einschaltung einer ausländischen Organisation zum Studium eines Projekts und Definition der wichtigsten Bedürfnisse - was natürlich in enger $\mathrm{Zu}$ sammenarbeit mit der Bevölkerung zu geschehen hat - können solche systeminhärenten Blockierungen oft sehr elegant umgangen werden. Die mit der Bevölkerung zusammen erarbeiteten Resultate gilt es dann in Verbindung zu setzen mit den Entwicklungszielen der Regierung sowie allenfalls den gesetzlichen Vorschriften der Entwicklungshilfeorganisa- 
tion. Die sich dabei herausschälende «Plateforme minimale de cohérence» zwischen Wünschen der Bevölkerung, Absichten der Regierung und Vorschriften der Hilfsorganisation bildet dann das Basisgerüst für die auszuführende Aktion.

Für Kamerun und Tschad kann die entsprechende «Landesprogramm-Plattform» vereinfacht wie folgt definiert werden:

\section{Kamerun: (Wald und Savannenregion)}

- Verbesserung und Diversifizierung der Nahrungsmittelproduktion (agrotechnische Aspekte)

- Vergrößerung der Einflußnahme des Bauern auf die Vermarktung der Nahrungsmittel; Organisierung der Bauern zwecks Verteidigung ihrer Interessen (sozio-ökonomische Aspekte)

- Die zu erwartenden Resultate (größere Geldmittel; politische Einflußmöglichkeiten) sollten eine wesentlich bessere Befriedigung der Grundbedürfnisse ermöglichen (z. B. Schule, Häuser «en dur», Dorfapotheken, Ganzjahres-Straßenverbindung usw.).

Tschad: (südliche Regionen: Savanne und Steppe)

- Sicherstellung der Selbstversorgung mit Nahrungsmitteln unter Wahrung des ökologischen Gleichgewichts, durch

- Verbesserung der Produktion durch angepaßtere Produktionsmittel (z. B. Einführung des Ochsenzugs) und angepaßtere Anbaumethoden (Pflügen; Mischkulturen usw.)

- Förderung von Maßnahmen zum Kampf gegen die Erosion (Niveaulinien) und zur Erhaltung der Bodenfruchtbarkeit (Gründüngung, Acacia albida)

- Verbesserung der Situation der Bauern im lokalen Vermarktungssystem (marché autogéré), was ihm die finanziellen Mittel gibt zu einer besseren Befriedigung einiger Grundbedürfnisse.

\section{Naturräumliche Grundlagen}

Zur Realisierung der in der «Plattform» definierten Ziele gilt es Lösungen zu finden, die den entsprechenden naturräumlichen Gegebenheiten angepaßt sind. Für Kamerun und Tschad, mit 70 bis $90 \%$ der Bevölkerung im primären Sektor (Kleinbauern) und der Forderung nach einer möglichst weitgehenden Selbstversorgung bedeutet dies, daß die Projekte vor allem eine möglichst optimale Inwertsetzung der landwirtschaftlichen Ressourcen anzustreben haben. Dabei gilt es insbesondere im Sahel darauf zu achten, daß der Erhaltung des ökologischen Gleichgewichts oder mindestens der Stabilisierung der jetzigen Situation größtes Gewicht zugemessen wird.

Diese Beachtung der naturräumlichen Grundlagen und die entsprechende Anpassung der Projektarbeit ist vorerst einmal eine «technische» Aufgabe. Wis- senschaftliche Untersuchungen dienen denn auch dazu, vor Projektbeginn die notwendigen Grundkenntnisse zu erarbeiten. Solche Untersuchungen sind jedoch äußerst zeitaufwendig und stoßen bei Bauern und Behörden, die möglichst rasch konkrete Resultate sehen möchten, auf immer weniger Verständnis. Sie werden deshalb üblicherweise nur dort durchgeführt, wo es um eine großräumige Erfassung eines Problems.(z. B. Desertifikationsdynamik im Sahel) oder die Vulgarisierung neuer und fundamentaler Innovationen geht. So war z. B bis vor kurzem die Großviehhaltung in der sudano-sahelischen Zone mit 800 bis $1000 \mathrm{~mm}$ Niederschlag/Jahr wegen der Tse-Tse-Fliege nur beschränkt möglich. Mit der Einführung neuer Methoden und Mittel und dem durch die Trockenheit bedingten Ausweichen von Nomaden gegen den Süden erfährt jetzt aber auch dieser Raum eine verstärkte Belastung, was den Anbau von Futtermitteln für das Großvieh nötig macht. In einem FAO/DEH-Projekt werden deshalb mit einem wissenschaftlichen Forschungs- und praktischen Anwendungsprogramm die geeignetsten Futterpflanzen bestimmt und anschließend in den entsprechenden Regionen des Sahelraums verbreitet.

Damit ist auch ausgedrückt, daß wissenschaftliche Untersuchungen im Rahmen der Entwicklungszusammenarbeit nicht zum Selbstzweck werden dürfen. Unser Ziel ist ja, Projekte zu realisieren. Eine Aktion kann sich also nicht in der Bereitstellung von Grundlagenmaterial erschöpfen. Vielmehr geht es um den Schritt hin zur konkreten Applikation der Erkenntnisse zwecks nachhaltiger Verbesserung der Situation der Bevölkerung.

Ein erfolgversprechendes und rascher zu realisierendes Vorgehen zur Berücksichtigung der natürlichen Faktoren besteht oft darin, die alten Kenntnisse der Bevölkerung wieder aufzunehmen und evtl. zu verbessern. Sie sind meist aufgrund jahrtausende alter Erfahrungen in ganz hervorragendem $\mathrm{Ma} \beta$ an die natürlichen Gegebenheiten angepaßt. Leider wurden diese lokalen Errungenschaften während der Kolonisation und auch später häufig als wenig seriös betrachtet und abgelehnt. In der Bevölkerung lebten diese traditionellen Verhaltensweisen und Arbeitsmethoden jedoch weiter. Die interessantesten davon in die Projekte einzubauen und weiterzuentwickeln bringt nicht nur im landwirtschaftlichen und ökologischen Bereich Vorteile, sondern auch in bezug auf die Partizipation der Bevölkerung in der betreffenden Aktion.

Als Beispiel sei die Acacia albida erwähnt, eine Akazienart, die wesentlich zur Erhaltung der Bodenfruchtbarkeit beiträgt. Die Bauern kennen diese Vorteile und bezeichnen diese Akazie in gewissen Gegenden des Tschad als «Lebensbaum». Sie haben sich bisher jedoch nie um Neupflanzung und Pflege der Bäume gekümmert. Mit der heutigen größeren Bestoßung der Regionen durch Klein- und Großvieh 
und den jährlichen Buschfeuern werden nun aber sozusagen alle Jungpflanzen zerstört. Es gilt deshalb, gemeinsam mit der Bevölkerung, Lösungen zu deren Schutz zu suchen und damit zur Erhaltung der Bodenfruchtbarkeit beizutragen. Dank den traditionellen Kenntnissen der Bauern und ihrem Interesse waren in diesem Bereich im Südtschad erstaunlich positive Resultate zu verzeichnen.

\section{Berücksichtigung der bestehenden Beziehungsgefüge}

Eine Entwicklungszusammenarbeit kann natürlich nicht losgelöst von den bereits bestehenden Strukturen und Aktivitäten realisiert werden. Die ermittelten Grundbedürfnisse, die aufgrund der naturräumlichen Voraussetzungen möglichen Aktionen, sind in Beziehung zu setzen mit dem gesamten bereits bestehenden Gefüge in den verschiedensten Bereichen. So spielen neben den "technischen» vor allem die sozio-ökonomischen und kulturellen Strukturen und Aktionsweisen eine ganz wichtige Rolle in bezug auf die langfristigen Erfolge und Auswirkungen von Entwicklungsprojekten.

Lange Zeit wurde diese Komplexität des Entwicklungsprozesses viel zu wenig beachtet; der Ansatz war zu punktuell ausgerichtet, zu stark auf das einzelne Projekt bezogen. Zusammenhänge wurden nicht erkannt oder konnten aufgrund mangelnder Erfahrungen nicht durchschaut werden.

Klassisches Beispiel sind die an sich positiven Verbesserungen im medizinischen Bereich, die u. a. zu einer starken Erhöhung der Überlebenschance der Kinder führten. Langfristig ergibt sich jedoch damit eine Bevölkerungszunahme, die zu einer Überbeanspruchung der Ressourcen und damit Zerstörung der Lebensgrundlagen führen kann. Dieser Prozeß spielt sich in bestimmten Gebieten des Sahelraumes $a b$, verstärkt noch durch die Trockenheit und eine veterinärmedizinisch bedingte Zunahme des Großviehs.

In einem unserer Projekte (vgl. dazu auch Anhang II) haben wir im Laufe der Realisierung lernen müssen, daß mit der Einführung des Ochsenzugs einerseits die übliche Brachzeit nicht mehr eingehalten werden konnte und damit die Bodenfruchtbarkeit zurückging, anderseits tiefgreifende Auswirkungen auf das soziale Gefüge in den Dörfern zu verzeichnen waren (Tendenz zur Privatisierung des Bodens, soziale Umstrukturierungen usw.). Beide Auswirkungen zwangen uns zu weitgehenden Projektanpassungen.

Es geht also darum, so weit wie möglich bereits vor dem Projektbeginn die Zusammenhänge zu erkennen, in welche sich das Projekt eingliedert, sowie alle Maßnahmen und Aktionen vorzusehen, um die negativen Auswirkungen zu minimisieren und die Par- tizipation der Bevölkerung sicherzustellen. Dazu ist folgendes zu bemerken:

a) Für die Gesamtschau, das Erkennen der Zusammenhänge bestehen noch relativ wenig Erfahrungen; die gesamte Problematik steht vielerorts in Bearbeitung (vgl.u.a.Programm MAB), mit Modellen, die eine Erfassung und Inbeziehungsetzung der Elemente erlauben sollen. Aufgrund konkreter Erfahrungen in Zentralafrika scheint es jedoch schwierig, im Rahmen von generalisierenden Modellen die regionalen Differenzen und lokalen Partikularitäten erfassen zu können.

Im praktischen Vorgehen haben wir vielmehr versucht, Spezialisten verschiedenster Bereiche, aber mit langer Erfahrung in der Entwicklungsarbeit, mit lokalen Mitarbeitern und Vertretern der Bauern, die eine gewisse Übersicht haben und die Reaktionen der Bevölkerung beurteilen können, zu einer «interdisziplinären» Gruppe zusammenzustellen und mit der Ausarbeitung der Zusammenhänge zu beauftragen. Dem Dialog und der Intuition kommen dabei größtes Gewicht zu. Dieses Vorgehen führte im Mikrobereich der Projektgebiete zu wesentlich brauchbareren Resultaten als die Arbeit mit Modellen.

Für die Darstellung regionaler Zusammenhänge anderseits ist eine modellartige Abstraktion nicht zu umgehen. Der Versuch einer Darstellung eines solchen regionalen Beziehungsgefüges (Sahelraum während der Trockenperiode 1969 bis 1974) ist im Anhang I aufgeführt.

b) Um in der praktischen Arbeit mit der Bevölkerung zu möglichst positiven und akzeptierten Entwicklungen zu gelangen, müssen wir uns von der Idee lösen, die Probleme kettenartig Punkt für Punkt anzugehen. Die Auswirkungen eines Eingriffs in einem bestimmten Bereich auf benachbarte Gebiete und die entsprechenden Rückkoppelungen zeigen, daß in vernetzten Strukturen oft gleichzeitig, mindestens jedoch überlappend verschiedene Aktionen durchzuführen sind. Nur mit dieser Art integrierter Projekte, die die wichtigsten zusammenhängenden Aspekte berührt, ist es möglich, zu einer einigermaßen gleichgewichtigen Entwicklung zu gelangen.

Das bereits erwähnte, in Anhang II dargelegte Beispiel eines integrierten Projekts zeigt auf, daß landwirtschaftliche Innovationen nicht nur Auswirkungen auf die Produktion haben, sondern gleichzeitig die natürlichen Ressourcen stärker beanspruchen, die Grundbedürfnisse verschieben, eine stärkere Integration des Bauern in die Marktwirtschaft fördern und schließlich zu mannigfachen sozialen Änderungen im engeren Lebensbereich führen. Bei all diesen Punkten gilt es, entweder Gegenmaßnahmen zu treffen oder die Bevölkerung soweit zu sensibilisieren und auszu- 
bilden, daß sie die Änderung verarbeiten und in ihre Lebensweise integrieren kann. Entsprechend sind bereits in den Projektansätzen, neben den Hauptaktionslinien, die notwendigen Begleitmaßnahmen vorzusehen, d.h. eben integrierte Projekte zu entwickeln.

\section{Partizipation der Bevölkerung}

Determinierender Faktor, ob ein Projekt überhaupt zu positiven Resultaten führen kann, ist die Mitarbeit der Bevölkerung in der Entwicklungsaktion, resp. die Integration der durch die Aktion bewirkten Innovationen in die Lebensbereiche der Bevölkerung. Ohne eine solche Partizipation ist eine Aktion langfristig sinnlos; sie wird nach Abzug der äußeren finanziellen, materiellen und personellen Mittel zusammenbrechen; eine Entwicklungsleiche mehr.

Die Landesprogramme und Aktionen sind deshalb so auszugestalten, daß mindestens die Voraussetzung für eine Mitarbeit der Bevölkerung geschaffen werden kann, z. B. durch Vermeidung einer zu starken Beeinflussung, resp. Beherrschung des Projekts durch die äußere Hilfe oder die ausführenden staatlichen Stellen. Die Schaffung und Durchsetzung von Mechanismen, die der betroffenen Bevölkerung nicht nur ein Mitsprache- sondern auch ein Mitbestimmungsrecht sichern, ist jedoch in der praktischen Projekttätigkeit mit erheblichen Schwierigkeiten verbunden. So ist eine Durchführung einer Aktion ohne ständige Berücksichtigung der Bevölkerungswünsche wesentlich einfacher und rascher zu bewerkstelligen und, kurzfristig gesehen, mindestens quantitativ erfolgreicher. Zudem wünschen die Behörden meist gar keine Strukturierung und Verantwortungsübernahme durch die Bevölkerung, ergäben sich doch dadurch oft wesentliche Änderungen in den herrschenden lokalen Machtverhältnissen. Diese Tatsache führt dann häufig zur ungewöhnlichen Situation, daß die Hilfsorganisation versuchen muß, die wirklichen Interessen der Bauern gegenüber den Behörden des Landes durchzusetzen. Daraus ergibt sich eine prinzipiell gar nicht erwünschte Verantwortungsübernahme durch den Geldgeber und eine mangelnde Identifikation der Behörden, was oft zu einer Blockierung des Projekts führen kann.

Ein anderes wichtiges Element in beżug auf die Partizipation ist die von einem ganz anderen soziokulturellen Hintergrund als dem unseren ausgehende Reaktionsweise der Bevölkerung. Die lokalen Werte, Mentalität und Denken führen oft zu anderen Entscheiden als wir Europäer (und häufig auch die Behörden) uns das vorgestellt haben.

So stieß z. B. im Tschad die von den Behörden geplante und von der DEH unterstützte Schulreform die u. a. eine Einführung praktischer landwirtschaft- licher Produktionsarbeiten vorsah - sowohl bei Lehrern wie Eltern und Schülern auf großen Widerstand. Größerer physischer Arbeitsaufwand, «akademischer» Niveauverlust der Schule durch Handarbeit, gefährdete soziale Stellung der Lehrer, enttäuschte Erwartungen der Eltern und Schüler und viele andere Faktoren verbanden sich zu einem Bündel an Wirkkräften, das nur teilweise rational erfaßt werden konnte, uns jedoch zum Abbruch der Aktion zwang.

Auch die Zuteilung eines Universitätsstipendiums und das Bestehen von Examen beruht nur teilweise auf den sonst üblichen Kriterien. Ethnische Zugehörigkeit, Stellung der Familie, Beziehungen zur Partei, politische Einstellung, Opportunität der Integration der entsprechenden Person in eine elitäre Schicht usw. sind Elemente, die schlußendlich wesentlich wichtiger sind als die Leistungen des Schülers.

Da sich Entwicklungsprojekte im Rahmen solcher Strukturen und Verhaltensweisen abzuspielen haben, ist deren Berücksichtigung im Rahmen eines Entwicklungsprogramms unentbehrlich, d. h. die Programme sind so flexibel zu halten, daß eine Anpassung an die Reaktionen der Bevölkerung jederzeit durchführbar ist.

Diese Reaktionen vorgängig zu definieren ist nur beschränkt möglich. Eine mehrjährige Arbeit auf dem Terrain jedoch kann dazu führen, daß man zu spüren beginnt, wie die Bauern (und Behörden) entscheiden und reagieren werden. Die Integration solcher Erfahrungswerte in ein Programm erleichtert natürlich eine Partizipation der Bevölkerung an dessen Realisierung und damit auch die erwünschte Übernahme der Aktion durch die Bevölkerung selbst.

\section{Zusammenfassung}

Die Ausarbeitung und vor allem die Ausführung eines «Landesprogramms» der Entwicklungszusammenarbeit in einem Land der Dritten Welt stellt eine äußerst vielfältige und komplexe Aufgabe dar, ein Ineinandergreifen mannigfacher Einflüsse und Faktoren.

Ein Teil davon - Definition der Bedürfnisse, planerische und technisch-wissenschaftliche Aspekte kann vor der Realisierung des Programms analysiert und definiert werden. Andere Aspekte, wie z. B. politische Einflüsse und Entscheide sowie Reaktionen der Bevölkerung, sind jedoch für uns Europäer nur teilweise logisch-rational durchschaubar. Profunde Kenntnisse der lokalen Verhältnisse gestatten aber oft ein intuitives Erfassen der Situation.

Um zu einer wirklichen Entwicklungszusammenarbeit zu gelangen und zu einer effektiven Verbesserung der Lebensbedingungen und Förderung der Ei- 


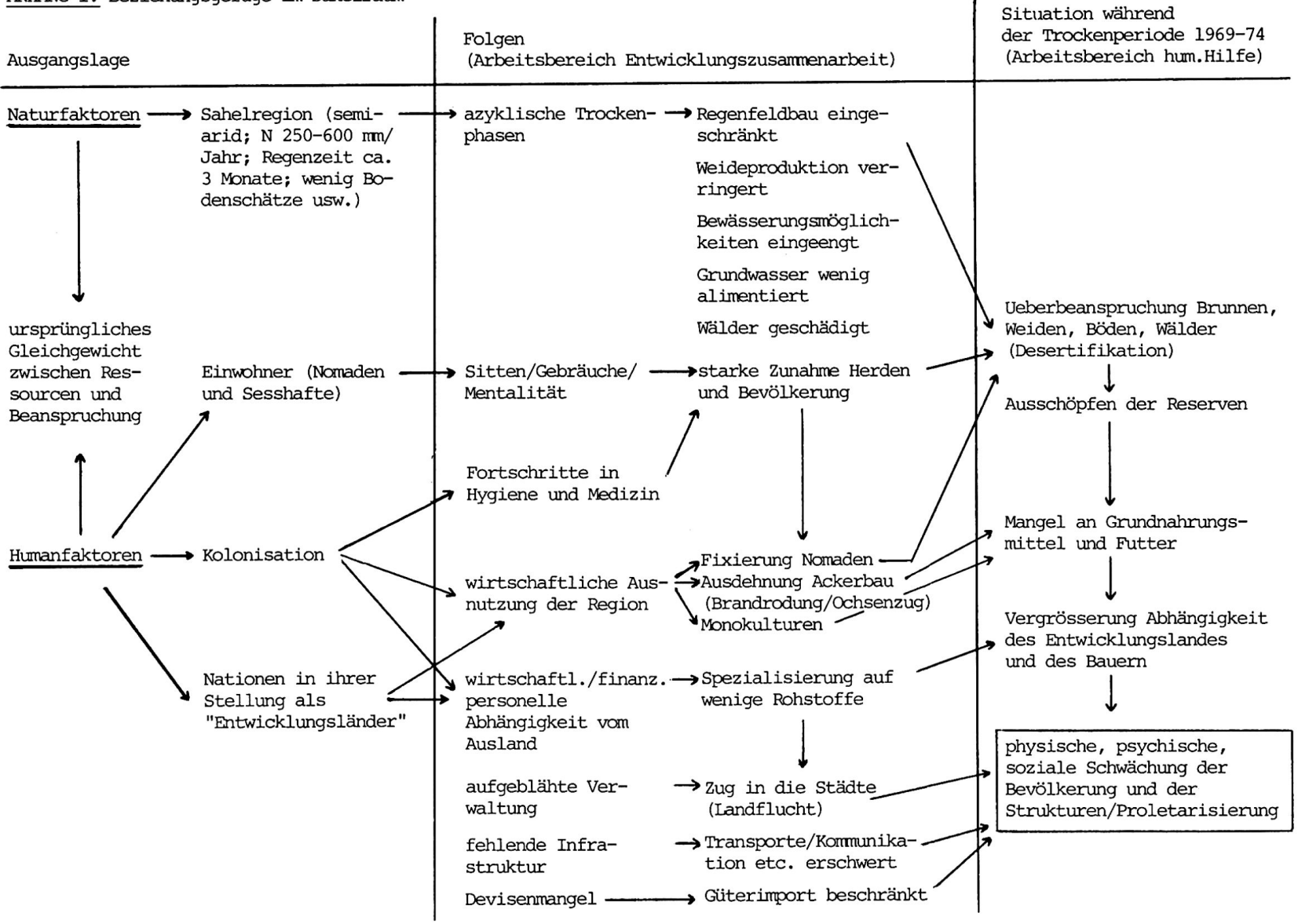


genverantwortung beizutragen, ist eine Mitarbeit der Bevölkerung in den Projekten notwendig. Dies ist nur erreichbar, wenn ihren Aspirationen und Reaktionen auch Rechnung getragen wird. Es gilt deshalb, die «definierbaren» Aspekte mit den eher «erfühlbaren» menschlichen und politischen Reaktionsweisen zu verbinden, um zu angepaßten Lösungen zu gelangen. Nur eine solche Zusammenfassung aller relevanten Faktoren kann zu angepaßten Landesprogrammen führen und damit als Grundlage dienen für unsere Unterstützung im betreffenden Entwicklungsland.

Die Geographie kann meiner Ansicht nach für die

\section{Anhang II}

Die Centres de formation professionnelle agricole, ein Beispiel eines integrierten ländlichen Entwicklungsprojektes im Südtschad.

\section{Allgemeiner Projektrahmen}

Zur Förderung des Hirseanbaus, aber auch zur Produktion von Exportprodukten wie Baumwolle und Erdnüsse, hat der Tschad seit rund 18 Jahren landwirtschaftliche Schulungszentren in Betrieb, die den Bauern mit neuen landwirtschaftlichen Techniken vertraut machen sollen (Einführung Ochsenzug, Einsatz Düngemittel und Pestizide, Mischkulturen usw.).

Die Ausbildungsmethode in diesen Centres de formation professionnelle agricole (CFPA) beruht auf folgenden Ideen:

- die Ausbildung darf den Bauern nicht von seinem Lebens- und Wirtschaftskreis entfremden; sie erfolgt deshalb in der Nähe seines Wohnortes, wohin er auch häufig während der Ausbildungszeit zurückkehrt. Ausgebildet werden nur Bauernfamilien (die Frau und die Kinder werden ebenfalls ins Zentrum aufgenommen). Die Ausbildung dauert ein Jahr (entspricht einer Vegetationsperiode) und ist auf die lokalen Produkte (Hirse, Baumwolle, Erdnüsse) ausgerichtet;

- die einzuführenden landwirtschaftlichen Neuerungen müssen dem Druchschnittsniveau des Bauern angepaßt und wirtschaftlich rentabel sein;

- neue landwirtschaftliche Methoden können nur über Bauern, die die lokalen Gemeinschaften vertreten, verbreitet werden. Deshalb wird von den Dörfern erwartet, daß sie bei der Auswahl der Stagiaires mitwirken;

- um keine Geschenke zu machen, wird von den Stagiaires für die Aufnahme ins Zentrum die Ro-
Ausarbeitung solcher grundsätzlicher Dokumente eine gute Ausgangsbasis bilden, sofern es ihr gelingt, die Analyse der naturräumlichen Gegebenheiten mit den vom Menschen ausgehenden Umgestaltungsmöglichkeiten zu verbinden. Dieses Zusammenführen der natürlichen und humanen Aspekte ist wohl das optimalste Vorgehen zur Erreichung einer langfristig positiven Entwicklung. Es wäre deshalb zu wünschen, daß sich die geographischen Institute, die sich ja recht weit entweder auf die naturwissenschaftliche oder auf die humangeographische Seite spezialisiert haben, verstärkt diesem Zusammenwirken öffnen könnten.

dung eines halben ha-Terrains sowie die Mitnahme der Nahrungsmittel für das Ausbildungsjahr verlangt.

Aufgebaut auf diesen Grundideen hat die DEH seit 1964 im Moyen Chari, im Süden des Tschad, bei der Schaffung von CFPA und Ausbildung der Bauern mitgewirkt.

\section{Resultate der landwirtschaftstechnischen Ausbildung}

Die wichtigsten Auswirkungen der Projektarbeit können wie folgt zusammengefaßt werden:

a) Von 1964 bis 1979 konnten rund 1600 Familien aus ca. 250 Dörfern ( = ca. $35 \%$ der im Gebiet der Zentren liegenden Dörfer) ausgebildet werden. 136 Bauernfamilien befanden sich 1980 in den 9 von der DEH aufgebauten Zentren in Ausbildung; pro Zentrum können jährlich maximal 24 Familien aufgenommen werden.

b) Produktivitätssteigerung: Je nach Zentrum ist eine Verdoppelung bis Verdreifachung der Produktion im Vergleich zu den durchschnittlichen Erträgen in den Dörfern festzustellen, und zwar sowohl für Baumwolle wie Hirse oder Erdnüsse. Nach der Rückkehr ins Dorf produziert der Bauer ca. doppelt so viel wie vor seiner Ausbildung.

c) Arbeitsmaterial: Der Verkauf der während des Ausbildungsjahrs erzielten Eigenproduktion gestattet den Stagiaires, sich das nötige Material (2 Ochsen, Pflug) zur Anwendung der neuen Methoden anzuschaffen.

d) Weitere Resultate der Ausbildung sind nicht chiffrierbar, haben jedoch einen großen Einfluß auf die Lebensweise der Familien, wie z. B größeres Einkommen, Reservebildung, bessere Zusammenarbeit in der Familie (u. a. auch durch Aus- 
bildung der Frauen), größeres Ansehen im Dorf usw.

Auch die Tatsache, daß die Zentren trotz den materiell recht belastenden Aufnahmebedingungen mehr Anmeldungen erhalten, als sie Stagiaires aufnehmen können, ist ein Hinweis auf eine positive Beurteilung ihrer Tätigkeit durch die Bauern.

e) Bodenfruchtbarkeit: Die Arbeit mit dem Pflug hatte einen gewissen Rückgang der Bodenfruchtbarkeit zur Folge. Gegenmaßnahmen (Gründüngung, Pflanzen von Acacia albida) werden jedoch erst nach einigen Jahren eine verbessernde Wirkung haben.

f) Arbeit mit Dorfgemeinschaften: Im Gegensatz zu den eher positiven landwirtschaftstechnischen Resultaten waren diejenigen im "sozialen Bereich» bescheiden oder sogar negativ: Die «Technisierung» der Arbeit erforderte eine teilweise Änderung des Anbau/Brachzyklus und förderte damit unerwünschte soziale Spannungen sowie Bodenprivatisierungsbestrebungen. Die Ausbildung im Zentrum initierte keine neuen Dorfaktivitäten und es entstand der Eindruck, daß die Zentren nur einer Kapitalbildung dienen.

Um diesen Mängeln Rechnung zu tragen, wurde seit 1974 der «soziale Bereich» zu einem weiteren, langfristig zu verfolgenden und in den Dörfern selbst zu realisierenden Schwerpunkt ausgebaut. Das Projekt erhielt damit seine «integrierende» Dimension.

\section{Die «integrierte» Ausrichtung}

Diese neue Ausrichtung des Projekts kann mit den folgenden Zielsetzungen charakterisiert werden:

- Ausbildung von Bauernfamilien im technischen, sozialen und menschlichen Bereich (in den Zentren und in den Dörfern);

- Ausbildung von Kadern, Stagiaires und Dorfdelegierten im Hinblick auf die Ausführung von gemeinsamen Dorfaktivitäten;

- Unterstützung der Dörfer zur Schaffung von Strukturen, die die Verantwortung für die «Activités communautaires» übernehmen und die für ihren Dorfbereich (und später die Region) ein Entwicklungsprogramm ausarbeiten und realisieren;

- Ständige Anpassung der Ausbildungsprogramme unter Mitarbeit der betroffenen Bauern und Kader.

Praktisch gesehen gilt somit die Projektarbeit - neben sozial angepaßterer Ausbildung in den Zentren vor allem einer Unterstützung von Dorfaktionen, gemäß dem Motto: se mettre à l'écoute du village pour chercher ensemble des solutions. Einige solcher gemeinsam erarbeiteten Lösungen seien kurz erwähnt:
- Eigenvermarktung der Baumwolle: Dieser «marché autogéré» bildet die eigentliche «Schlüsselaktion» zur Realisierung des Dorfprogramms. Die Bauernfamilien lernen dabei ihre Baumwolle selbst zu wägen, die Gewichte zu addieren, den Wert ihrer Produkte auszurechnen und das entsprechende Geld abzuzählen. Wichtigste Resultate dieser Ausbildung in lokaler Sprache sind die Förderung des Vertrauens eines Dorfes in das eigene Können durch Beherrschung verschiedener Operationen, eine Ausschaltung der Ausbeutung der Bauern durch die bisherigen Baumwollaufkäufer, die Äufnung eines «Fonds villageois» sowie die Sammlung erster Erfahrungen in kollektiver Arbeit.

Bisher konnte in rund 100 Dörfern ein Marché autogéré durchgeführt werden; 1978/79 ergaben diese Märkte einen Umsatz von 2922000 kg Baumwolle. Zusätzlich zu dem den Bauern vollständig ausbezahlten Preis für ihre Produktion warf diese vermarktete Menge 3,6 Millionen CFA-«Prämien» ab, die nun als «Fonds villageois» für die Finanzierung weiterer Dorfaktionen verwendbar sind (d. h. pro Dorf im Durchschnitt 50000 CFA/400 sFr., was ca. zur Finanzierung von 1-2 weiteren Aktionen genügt).

Solche von den Dörfern selbst bestimmte weitere Dorfaktionen waren u. a.:

- Pharmacie de village: Haltung einer kleinen Apotheke mit Basismedikamenten, die der Bevölkerung verkauft werden. Ausbildung einer Equipe de gestion und des Verantwortlichen der Apotheke (Erkennen der wichtigsten Krankheiten, Hygiene usw.).

Diese Ausbildung ist nach den Marchés autogérés am meisten gefragt, insbesondere auch, da in diesem Fall vermehrt Frauen mitarbeiten können.

- Action d'élevage: Bau eines Impfgangs und einer Silagegrube, Lieferung von veterinärmedizinischem Material, Ausbildung der Verantwortlichen.

- Boutique d'approvisionnement: zur Versorgung der Dörfer mit den Grundgütern (Zucker, Salz, Petrol, Seife usw.).

- Bau von Gemeinschaftslokalen (Materialdepot, Versammlungsraum usw.).

- Brunnenbau.

- Verschiedene Spezialkurse für Frauenarbeiten (Nähen, Sticken, Färben), Schreinern, Einführung neuer landwirtschaftlicher Produkte usw.

Alle diese Kurse sind verbunden mit einer Alphabetisierung, die speziell auf die Vermittlung der für das entsprechende Sachgebiet notwendigen Kenntnisse ausgerichtet ist (in lokaler Sprache). Je nach Fall wird diese funktionelle Alphabetisierung erweitert, um der Bevölkerung Grundkenntnisse mindestens in Lesen und Rechnen zu geben. Zur Unterstützung 
und vor allem Erhaltung dieses Wissens erscheint 2bis $3 \mathrm{mal}$ pro Jahr eine Zeitschrift, die neben allgemeinen und landwirtschaftstechnischen Beiträgen vor allem Mitteilungen aus den Zentren und über Dorfaktionen sowie erstmals kulturelle Artikel (Dorferzählungen, Sagen usw.) veröffentlicht.

Zur Realisierung dieser Aktion ist natürlich ein gut ausgebildetes und motiviertes Kader notwendig; da liegt denn auch eine der großen Schwierigkeiten. Um die Kader vermehrt auf eine Berücksichtigung der Wünsche der Bevölkerung auszurichten, wurde die Ausbildung mehr und mehr von rein agrotechnischen Themen auf ein die wichtigsten Aspekte des ländlichen Milieus beachtendes Programm erweitert (Alphabetisierung, Aufbau und Führung einer Kooperative, pädagogisches Vorgehen bei der Dorfarbeit, Animation und Sensibilisierung usw.).

Ein weiterer wichtiger Aspekt betrifft den Einbezug der Frau in die Entwicklung. Angesichts der mannigfachen Verantwortung der afrikanischen Frau für ihre Familie (z. B. Nahrungsmittelbeschaffung) wurde neben Education sanitaire, Kinderpflege usw. vor allem versucht, für die Frau neue Einnahmequellen zu finden, damit die bisherige Tätigkeit um Geld zu verdienen, nämlich die Herstellung von Hirsebier, etwas eingeschränkt werden kann. Anhand eines "Carnet de pharmacie» ist ebenfalls eine funktionelle Alphabetisierung der Frauen in lokaler Sprache möglich.

\section{Literaturbesprechung}

KUKLINSKY Antoni (Hrsg.): Growth Poles and Growth Centres in Regional Planning. The Hague, Mouton, 1972, 306 S., Fr. 45.--.

STOEHR Walter: Regional Development Experiences and Prospects in Latin America. The Hague, Mouton, 1975, $188 \mathrm{~S}, \mathrm{Fr}$. 36.--.

Mitte der sechziger Jahre beschlossen die United Nations Organisations ein Forschungsprogramm zum Thema "Regionalentwicklung" aufzuziehen.

Mit der Konzeption und Durchführung dieses Programms wurde das United Nations Research Institute for Social Development (UNRISD) mit Sitz in Genf betraut. Die Leitung dieses Programms wurde Antoni KUKLINSKY, einem polnischen Wirtschaftsgeographen, übertragen, dem in den ersten Programmjahren Tormod HERMANSEN, ein norwegischer Oekonom, zur Seite stand. Beide Wissenschaftler leisteten im Rahmen dieses Programmes entscheidende Beiträge zur Entwicklungsforschung.

Zur Zeit wird dem "Regional Development Programme" auch auf internationaler Ebene leider

\section{Zusammenfassung}

In der 18jährigen Arbeit im vorliegenden Projekt wird ein langer Entwicklungsprozeß sichtbar: Ausgangspunkt ist eine zentralisierte, agrotechnische Ausbildung, die zu technisch positiven Resultaten führte. Das Vorgehen konnte jedoch im soziokulturellen und ökologischen Bereich nicht befriedigen und verhinderte eine eingehende Partizipation der Bevölkerung. Die Auslagerung der Tätigkeit in die Dörfer und die Konzentration auf Aktionen, die von den Dörfern selbst ausgewählt, bezahlt, aber auch kontrolliert werden, führte zu einer verstärkteren Verankerung des Projekts bei der Bevölkerung und damit zu wesentlich größeren Auswirkungen auf die Aktivitäten der Bauern. Bei diesem Erfolg gilt es jedoch zu beachten, daß nur aufgrund einer seriösen landwirtschaftlichen Ausbildung das Vertrauen der Bevölkerung und auch der verantwortlichen staatlichen Organe gewonnen werden konnte, um neben den landwirtschafts-technischen auch im weitesten Sinne soziale Aktionen realisieren zu können und damit zu einem wirklich integrierten Projekt zu gelangen, das auf Bewußtseinsförderung, Eigenverantwortung und schlußendlich Kontrolle der Entwicklung durch die Bevölkerung selbst hinzielt. Die DEH hofft, an diesem sozio-ökonomischen Entwicklungsprozeß, der mehrere Jahre dauern dürfte, weiterhin aktiv mitwirken zu können.

nur noch wenig Beachtung geschenkt. Dies ist umso mehr zu bedauern, als die Probleme regional ungleichgewichtiger Entwicklung auf allen räumlichen Massstabsebenen nicht kleiner geworden sind, sondern gerade in den letzten Jahren an Brisanz noch gewonnen haben.

Aus dem UNRISD-Forschungsprogramm resultierte eine Reihe srundlegender Publikationen namhafter Autoren. Diese Arbeiten können in drei Gruppen eingeteilt werden:

- Länderstudien (LEFEBER \& DATTA CHAUDURI 1971, MILHAILOVIC 1975, STOEHR 1975, KUKLINSKY 1978)

- Theoretische Arbeiten z.B. über die Bedeutung von Wachstumspolkonzepten oder Informationssystemen für regionale Entwicklungsprozesse (KUKLINSKY 1972, PEDERSEN 1974)

- Methodische Studien über Planungstechniken (KUKLINSKY 1974)

Der hier zu besprechende Sammelband "Growth Poles and Growth Centres in Regional Planning" gehört in die Gruppe theoretischer Arbeiten und 